\title{
DISEÑO Y VALIDACIÓN DE UN INSTRUMENTO DE AUTOEVALUACIÓN DEL ALUMNADO UNIVERSITARIO DENTRO DEL MODELO FLIPPED \\ LEARNING
}

Design and validation of a self-assessment instrument of the university student in the flipped learning model

Desenho e validação de um instrumento de auto-avaliação de alunos no modelo flipped learning

\section{Fernando M. Otero-Saborido (1)}

Antonio Sánchez-Oliver (2)

\section{Inmaculada Fuentes-García (3)}

(1) Universidad Pablo de Olavide de Sevilla, España. Teléfono: 954977 961. Correo electrónico: fmotero@upo.es

(2) Universidad Pablo de Olavide de Sevilla, España. Correo electrónico: asanchez@upo.es

(3) Universidad Pablo de Olavide de Sevilla, España. Correo electrónico: ifuegar@upo.es

\section{Resumen}

El imperio de las metodologías tradicionales es una realidad en la enseñanza universitaria. Por ello, el objetivo de este trabajo fue el diseño y validación de un instrumento de autoevaluación continua que implicara al alumnado universitario en procesos de reflexión sobre su aprendizaje en el modelo Flipped Learning. Para ello, 84 alumnos (18.88 \pm 1.18$)$ de primer curso del Grado de Ciencias de la Actividad Física y del Deporte participaron durante 12 semanas en la cumplimentación semanal de un instrumento de autoevaluación. El cuestionario siguió una validación de contenido por un grupo de expertos y, posteriormente, se halló la confiabilidad desde la perspectiva consistencia interna a través del Alpha de Cronbach. Los resultados obtenidos muestran instrumento fiable que facilita el trabajo por competencias en la enseñanza universitaria bajo el modelo Flipped Learning.

Diseño y validación de un instrumento de autoevaluación del alumnado universitario dentro del Modelo 
Palabras clave: Aula invertida; metodologías activas; evaluación formativa

\begin{abstract}
The empire of traditional methodologies is a reality in university education. Therefore, the objective of this work was the design and validation of a continuous self-assessment instrument that involves university students in processes of reflection on their learning in the Flipped Learning model. To this end, 84 students $(18.88 \pm 1.18)$ of the first year of the Degree of Physical Activity and Sport Sciences participated for 12 weeks in the weekly completion of a self-assessment instrument. The questionnaire followed content validation by a group of experts and, subsequently, reliability was found from the internal consistency perspective through Cronbach's Alpha. The results obtained show a reliable instrument that facilitates the work by competences in university teaching under the Flipped Learning model.
\end{abstract}

Keywords: Flipped Classroom; Active methodologies; formative assessment

\title{
Resumo
}

A regra de metodologias tradicionais é uma realidade no ensino superior. Portanto, o objetivo deste trabalho foi a concepção e validação de um instrumento de auto-avaliação contínua que envolve estudantes universitários na reflexão sobre a sua aprendizagem no modelo Flipped Learning. Para fazer isso, 84 alunos $(18,88 \pm 1,18)$ Primeiro ano Bachelor of Science em Atividade Física e do Desporto participou durante 12 semanas no semanário completando uma ferramenta de auto-avaliação. A validação de conteúdo questionário seguido por um grupo de peritos e, em seguida, a dirigibilidade foi encontrada a partir da consistência interna perspectiva através alfa de Cronbach. Os resultados mostram ferramenta confiável que facilita o trabalho de competências no ensino superior sob o modelo Flipped Learning.

Palavras-chave: Classe para trás; metodologias activas; avaliação formativa

\section{Introducción}

La pedagogía tradicional ha puesto el foco en la enseñanza, no en el aprendizaje; ha centrado sus esfuerzos e investigaciones en el docente, no en el alumnado (Sicilia, 
2004). Esta situación dibuja dos rasgos en la enseñanza universitaria. Por una parte, confundir y entender la calificación académica como sinónimo de evaluación y, por otro, lo que se ha llamado "ficción de los ECTS" (European Credit Transfer and Accumulation System) (Gómez et al 2011; Prieto, 2014). Esta ficción señala una deficiencia entre los créditos otorgados a una asignatura (que integran la parte presencial y la no presencial) y la carga crediticia que en realidad desarrolla el alumnado en horario no presencial. En este sentido, el problema del déficit entre ECTS y horas reales no es sólo cuantitativo. Nos encontramos ante una cuestión de más calado. La “ficción” del trabajo no presencial dilapida la contribución de las ya manidas competencias al aprendizaje del alumnado universitario (Zabalza, 2004). Como respuesta a esa “ficción” los modelos de participación activa como Flipped Learning empoderan las competencias del alumnado y favorecen el planteamiento de actividad no presencial que plantea el sistema ECTS. El objetivo del presente trabajo fue el diseño y validación de un instrumento de autoevaluación continua que implicara al alumnado universitario en procesos de reflexión sobre su aprendizaje en el modelo Flipped Learning. Así mismo, se pretendía que dentro de estos procesos, el instrumento facilitara una estimación cualitativa y cuantitativa del trabajo presencial y no presencial realizado en la asignatura.

\section{Método}

Participantes. Un total de 84 alumnos $(18.88 \pm 1.18)$ del primer curso del Grado de Ciencias de la Actividad Física y del Deporte participaron en esta investigación. La recogida de información se realizó en el curso 2016-2017 en la Universidad Pablo de Olavide de Sevilla.

Procedimiento. Para el objetivo del presente trabajo se procedió a la validación completa del instrumento siguiendo dos fases: Una validación de contenido y la confiabilidad del instrumento. Para la validación de contenido un grupo de expertos determinó los objetivos de la herramienta: 1) Recoger la percepción sobre del alumnado sobre el tiempo de trabajo dedicado tanto a tareas presenciales como no presenciales. 2) Implicar al alumnado en la reflexión de los elementos del proceso de enseñanza aprendizaje. Posteriormente, se realizó una revisión bibliográfica en torno a dos ámbitos, por un lado, la evaluación formativa a través de la autoevaluación y, por otro, Diseño y validación de un instrumento de autoevaluación del alumnado universitario dentro del Modelo 
el modelo Flipped Learning. Dado el objetivo y el contexto a aplicar el instrumento, el grupo de expertos determinó que el instrumento final debía tener dos requisitos. En primer lugar, las variables/categorías no deberían circunscribirse únicamente al alumnado sino que contemplaran todos los agentes/elementos de la enseñanzaaprendizaje: docente, contenidos y/o proceso. En segundo lugar, se acordó que el instrumento final tuviera un reducido número de ítems dado que iba ser cumplimentado por el alumnado semanalmente a lo largo de un semestre. Tras un proceso de circulación del cuestionario, el instrumento inicial de 12 preguntas se redujo a 8 preguntas finales. Las dos primeras preguntas tienen un formato de respuesta abierto. Las preguntas 3,4 y 5 tienen una escala de tipo Likert con cuatro opciones de respuesta y una pregunta cualitativa cada una para justificar la respuesta. Posteriormente, el alumnado participante cumplimentó semanalmente el instrumento de evaluación diseñado por los expertos sumando un total de 12 cuestionarios por cada alumno.

Análisis. Para el análisis de la confiablidad se analizó la consistencia interna a través del Alpha de Cronbach.

\section{Resultados y discusión}

Los resultados obtenidos muestran coeficientes del Alpha de Cronbach buenos y aceptables en 12 de las 14 categorías del instrumento de autoevaluación (Tabla 1). Es cuestionable el coeficiente obtenido en la categoría 'EB' $(0,559)$ de la variable CAPA e inaceptable $(0,292)$ el de la categoría 'PP' de la variable CANPA. Por otra parte, en 11 de las 14 categorías la consistencia interna es muy significativa. La mayoría de los trabajos revisados sobre FlippedLearning no utilizan instrumentos de evaluación o los utilizados no han sido validados (Calvillo, 2014; Martín y Santiago, 2016; Perdomo, 2016). En el caso de instrumentos validados dentro del modelo Flipped no están dirigidos a valorar de forma continuada el trabajo no presencial del alumnado ni su implicación, sino más bien que el alumnado valore específicamente del modelo FlippedLearning (Flores, Del Arco y Silva, 2016). A pesar de que todos los coeficientes no son cuantitativamente buenos, el instrumento pueden considerarse globalmente pertinente si se tiene en cuenta el tamaño de la muestra $(n=84)$ y que los datos obtenidos no se obtienen para tomar decisiones experimentales posteriores. 
Tabla 1.

Consistencia interna de las variables y categorías del instrumento de autoevaluación

\begin{tabular}{|c|c|c|c|c|c|}
\hline Variable & \multirow{2}{*}{$\frac{\text { Categoría }}{\text { EB }}$} & \multirow{2}{*}{$\begin{array}{c}\begin{array}{c}n \\
\text { ítems }\end{array} \\
12\end{array}$} & \multirow{2}{*}{$\begin{array}{c}\begin{array}{c}\text { Alfa de } \\
\text { Cronbach }\end{array} \\
0,559\end{array}$} & \multirow{2}{*}{$\begin{array}{c}\mathbf{F} \\
, 017\end{array}$} & \multirow{2}{*}{$\begin{array}{c}\text { Sig. } \\
, 017^{* *}\end{array}$} \\
\hline \multirow{3}{*}{ CAPA } & & & & & \\
\hline & EP & 12 & 0,737 & 5,294 &, $000 * *$ \\
\hline & $\mathrm{TP}$ & 12 & 0,952 & 2,103 &, $018 * *$ \\
\hline \multirow{8}{*}{ 2. CANPA } & LT & 12 & 0,884 & 1,319 & 208 \\
\hline & VV & 12 & 0,692 & 48,342 &, $000 * *$ \\
\hline & TV & 12 & 0,623 & 2,880 &, $001 * *$ \\
\hline & $\mathrm{PP}$ & 12 & 0,292 & 14,181 &, $000 * *$ \\
\hline & $\mathrm{EE}$ & 12 & 0,743 & 74,926 &, $000 * *$ \\
\hline & $\mathrm{AE}$ & 12 & 0,862 & 1,520 & 118 \\
\hline & OT & 12 & 0,844 & 1,413 & 161 \\
\hline & $\mathrm{TN}$ & 12 & 0,882 & 9,168 &, $000 * *$ \\
\hline \multicolumn{2}{|c|}{ Implicación alumnado } & 12 & 0,822 & 3,406 &, $000 * *$ \\
\hline \multicolumn{2}{|c|}{ Utilidad aprendizajes } & 12 & 0,769 & 3,406 &, $000 * *$ \\
\hline 5. & cencia & 12 & 0,705 & 7,149 &, $000 * *$ \\
\hline
\end{tabular}

CAPA: Cantidad de Actividad Presencial del alumnado; EB: Enseñanza Básica; EP: Enseñanza Práctica: Enseñanza Práctica; TP: Tutoría Presencial; CANPA: Cantidad de Actividad No Presencial; LT: Lectura de Textos: LT; VV:Visionado de Videos; TV: Tutorías Virtuales; PP: Preparación de Práctica; EE: Estudio Examen; Autoevaluación: AU; OT: Otros TN: Total No Presencial. ${ }^{*} \mathrm{p}<0.05$; ** $\mathrm{p}<0.001$

\section{Conclusiones}

Este trabajo ha cumplido con el objetivo planteado de diseñar y validar un instrumento de autoevaluación continua que implicara al alumnado universitario en procesos de reflexión dentro del Modelo FlippedLearning. Así mismo, la herramienta facilita la estimación del trabajo no presencial del alumnado tan importante en el sistema ECTS y en la adquisición de competencias genéricas y específicas del alumnado universitario.

\section{Referencias}

Calvillo, A. J. (2014). El modelo Flipped Learning aplicado a la materia de música en el cuarto curso de Educación Secundaria Obligatoria: una investigación-acción para la mejora de la práctica docente y del rendimiento académico del alumnado. TesisDoctoral. Departamento de Pedagogía. Universidad de Valladolid.

Diseño y validación de un instrumento de autoevaluación del alumnado universitario dentro del Modelo 
Flores, O., Del Arco, I., \& Silva, P. (2016).The flipped classroom model at the university: analysis based on professors' and students' assessment in the educational field. International Journal of Educational Technology in Higher Education 13:21

Goméz, F., Barjola, P., Díaz, G., González, J.L., López, A., Mercado, F.J., \&Rivas, I. (2011). Cálculo del volumen de trabajo del alumno en las nuevas titulaciones de ciencias de la salud en Evaluación global de los resultados del aprendizaje en las titulaciones dentro del Espacio Europeo de Educación Superior / coord. Labrador, F. Santero, R. págs, 417-436

Martín, D., \& Santiago, R. (2016). Flipped Learning en la formación del profesorado de secundaria y bachillerato. Formación para el cambio. Contextos educativos, 1, 117-134.

Perdomo, W. (2016). Estudio de evidencias de aprendizaje significativo en un aula bajo el modelo Flipped Classroom. EDUTEC, Revista Electrónica de Tecnología Educativa, 55.

Prieto, A., Díaz, D., Monserrat, J., \& Reyes, E. (2014) Experiencias de aplicación de estrategias de gamificación a entornos de aprendizaje universitario ReVisión 7(2) pp. 76-92

Sicilia, A.(2003). La investigación sobre el pensamiento del alumnado. Una revisión desde la Educación Física. Revista de Educación,331, 577-613.

Zabalza, M.A. (2004) Trabajar por competencias: implicaciones para la práctica docente. Ponencia del Programa de Formación Permanente del Profesorado de la Universidad de Sevilla. Curso 2004-2005.

Diseño y validación de un instrumento de autoevaluación del alumnado universitario dentro del Modelo 